\title{
Avaliação Clínica e Microbiológica do Tratamento da Estomatite Protética com Tintura de Schinus terebinthifolius Raddi (Aroeira)
}

\author{
Clinical and Microbiological Evaluation of the Treatment of Denture Stomatitis with \\ Schinus terebinthifolius Raddi (Aroeira) Tincture
}

\author{
Diana Gabriela de Sousa SOARES ${ }^{1}$, Cibele Braga de OLIVEIRA², Marçal de Queiroz PAULO ${ }^{3}$, Maria de Fátima Farias Peixoto \\ CARVALHO ${ }^{4}$, Wilton Wilney Nascimento PADILHA ${ }^{5}$
}

${ }^{1}$ Doutoranda em Reabilitação Oral pela Faculdade de Odontologia da Universidade Estadual Paulista (UNESP), Araraquara/SP, Brasil. ${ }^{2}$ Mestranda em Ciências Odontológicas pela Faculdade de Odontologia da Universidade Estadual Paulista (UNESP), Araraquara/SP, Brasil. ${ }^{3}$ Professor Titular da Disciplina de Química do Departamento de Química da Universidade Federal da Paraíba (UFPB), João Pessoa/PB, Brasil. ${ }^{4}$ Bioquímica do Laboratório de Micologia do Departamento de Ciências Farmacêuticas da Universidade Federal da Paraíba (UFPB), João Pessoa/ PB, Brasil.

${ }^{5}$ Professor Doutor da Disciplina de Clínica Integrada do Departamento de Clínica e Odontologia Social da Universidade Federal da Paraíba (UFPB), João Pessoa/PB, Brasil.

\section{RESUIMO}

Objetivo: Avaliar a eficácia clínica da tintura da aroeira no tratamento da estomatite protética.

Método: Foram selecionados 18 pacientes usuários de próteses removíveis com diagnóstico clínico para estomatite protética tipo II e presença de candidose associada à prótese, constatados a partir de um exame clínico e micológico. Os pacientes foram distribuídos em dois grupos: GT (grupo teste) - tratamento com a tintura da aroeira; GC (grupo controle), tratamento com nistatina. Todos os pacientes foram orientados a higienizar a prótese com escova e dentifrício e, em seguida, aplicar o produto na mucosa palatina e na superfície da prótese 3 vezes ao dia, durante 15 dias consecutivos, remover a prótese à noite e armazená-la em um recipiente com água. No 15으 dia de uso, foi realizado um novo exame clínico e micológico para avaliar a eficácia do tratamento. Os dados foram analisados com os testes Wilcoxon e Mann-Whitney com nível de signficância de $5 \%$.

Resultados: Observou-se eliminação do processo inflamatório e da infecção por Candida spp. em $66,7 \%$ e $77,8 \%$ dos casos, respectivamente, para GT. Já para GC, a eliminação do processo inflamatório e da infecção fúngica ocorreu em 77,8\% e $88,9 \%$ dos casos, respectivamente. Estes resultados foram estatisticamente significantes (Wilcoxon $p=0,01$ ). Não foi observada diferença estatísticamente significante entre os dois tratamentos (Mann-Whitney $p>0,05$ ). A infecção fúngica foi diagnosticada apenas na prótese em todos os casos, sendo a C. albicans o microorganismo mais prevalente, estando presente em $94,4 \%$ dos casos

Conclusão: $O$ tratamento com a tintura da aroeira foi eficaz no tratamento da estomatite protética, promovendo remissão do processo inflamatório e da infecção por Candida spp.

DESCRITORES

Estomatite sob prótese; Ensaio clínico; Candida.

\section{ABSTRACT}

Objective: To evaluate the clinical efficacy of Schinus terebinthifolius Raddi (aroeira) tincture in the treatment of denture stomatitis.

Method: Eighteen removable denture wearers with clinical diagnosis of type II denture stomatitis and presence of candidosis associated to the denture use, as confirmed by clinical and mycological examinations, were selected for the study. The patients were allocated to two groups: TG (test group) - treatment with Schinus terebinthifolius Raddi (aroeira) tincture; CG (control group), treatment with nystatin. All patients were instructed to clean the dentures with toothbrush and dentifrice, and then apply the product on the palatal mucosa and on denture surface 3 times a day, during 15 consecutive days, removing the denture at bedtime and keeping it in a receptacle with water. At the 15 th day of use, the clinical and mycological examinations were redone to evaluate treatment efficacy. Data were analyzed statistically by Wilcoxon and Mann-Whitney tests at 5\% significance level.

Results: The inflammatory process and Candida spp. infection were eliminated in $66.7 \%$ and $77.8 \%$ of the cases, respectively, in TG. In CG, elimination of the inflammatory process and fungal infection occurred in $77.8 \%$ and $88.9 \%$ of the cases, respectively. These results were statistically significant $(p=0.01)$. There was no statistically significant difference between the treatments ( $p>0.05)$. In all cases, fungal infection was detected only on the denture, and $\mathrm{C}$. albicans was the most prevalent microorganism, being present in $94.4 \%$ of the cases.

Conclusion: The treatment with Schinus terebinthifolius Raddi (aroeira) tincture was effective in the treatment of denture stomatitis, promoting remission of the inflammatory process and Candida spp infection.

\section{KEYWORDS}

Stomatitis, denture; Clinical trial; Candida. 


\section{INTRODUÇÃO}

A candidíase oral, quando acomete pacientes portadores de próteses dentárias, é descrita como estomatite protética e tem sido considerada a lesão bucal mais freqüentemente observada em usuários de próteses removíveis, apresentando uma prevalência de 60 a $72 \%^{1,2}$. Clinicamente, esta lesão caracterizase pela presença de múltiplos pontos hiperêmicos na região palatina sob as próteses removíveis, e em casos mais avançados, também podem ser observadas áreas eritematosas difusas, ou ainda, hiperplasia papilar do palato $^{1,3}$.

A estomatite protética apresenta etiologia multifatorial, entretanto, o estabelecimento de um biofilme na base da prótese dentária é considerado como um dos principais fatores etiológicos. Este biofilme é composto por uma heterogenicidade de microorganismos, sendo os Streptococcus spp. e Candida spp. os mais prevalentes. Outras espécies presentes são Actinomyces spp., Lactobacillus spp. e Veillonella spp. Esta heterogenicidade do biofilme pode ter impacto na habilidade da infecção responder a terapia antimicrobiana, pois modificações da matriz do biofilme produzida pelos diversos microorganismos podem reduzir a penetração dos agentes antifúngicos ${ }^{4}$.

A C. albicans é a espécie fúngica mais comumente associada em pacientes com estomatite protética, sendo responsável por aproximadamente $70 \%$ dos casos de infecção $0^{1,2}$. O local preferencial de instalação deste microorganismo é a superfície interna da prótese, o que protege os microorganismos da ação de lavagem da cavidade oral, bem como da ação de substâncias antimicrobianas provenientes da saliva ${ }^{5,6}$. A presença da C. albicans na superfície interna da prótese acarreta numa reação inflamatória da mucosa palatina subjacente devido à atuação de suas enzimas sobre os tecidos, que provocam degradação tecidual pela liberação de enzimas hidrolíticas, promovendo uma resposta imune mediada por células ${ }^{7}$.

O tratamento tradicional da estomatite protética consiste em orientação do paciente para correta higienização da prótese dentária e utilização de antifúngicos tópicos, como a Nistatina e o Miconazol, e antifúngicos sistêmicos, como a Anfotericina B e o Fluconazol. Porém, a administração freqüente de doses relativamente elevadas requeridas na terapia podem ocasionar reações sistêmicas adversas e a recidiva é freqüente ${ }^{8}$. Neste contexto surgem os agentes fitoterápicos, que são uma alternativa terapêutica eficaz, de baixo custo e com efeitos colaterais diminuídos, sendo mais acessíveis à população.

A aroeira (Schinus terebonthifolius Raddi) é uma planta nativa da região nordeste do Brasil. Esta planta apresenta diversas propriedades farmacológicas, como atividade antimicrobiana, antiinflamatória e cicatrizante, as quais estão relacionadas com componentes químicos presentes em diferentes partes da planta, como taninos, flavonóides e triterpenos ${ }^{9}$. Devido a essas propriedades, a aroeira apresenta um amplo emprego dentro da medicina popular, o que permite sua escolha em estudos clínicos, farmacológicos e químicos, visando sua validação como medicamento eficaz e seguro ${ }^{10}$.

Portanto, neste estudo objetivou-se avaliar a eficácia da tintura da casca aroeira no tratamento da estomatite protética, analisando-se a remissão do processo inflamatório e da infecção por Candida spp. associada à prótese.

\section{METODOLOGIA}

Este trabalho foi aprovado pelo Comitê de Ética em Pesquisa do Centro de Ciências da Saúde da Universidade Federal da Paraíba (Protocolo no 1340), estando de acordo com os requisitos nacionais e a Declaração de Helsinki.

Realizou-se a a coleta da casca da aroeira (Schinus terebinthifolius Raddi), no período da manhã, no Campus I da Universidade Federal da Paraíba, na cidade de João Pessoa/PB.

A tintura da casca da aoreira foi realizada no Laboratório Químico e de Produtos Naturais da UFPB. As cascas do tronco da árvore foram retiradas e deixadas para secar ao ar livre. A tintura foi preparada através da maceração da casca da planta seca à temperatura ambiente, utilizando-se álcool de cereais como líquido extrator, correspondendo à $1 / 5$ do seu peso em erva seca, obtendo-se uma concentração final de 20\% (200 $\mu \mathrm{g} / \mathrm{mL}$ ). Em seguida, a tintura foi filtrada em papel filtro Whatman $\mathrm{n}$ ㅇ 01 e conservada sob refrigeração. A escolha desta concentração foi baseada em trabalho prévio ${ }^{9}$, o qual observou atividade do extrato alcoólico da casca da aroeira frente à C. albicans até a concentração de 1250 $\mu \mathrm{g} / \mathrm{mL}$

Cada $100 \mathrm{ml}$ do spray continha $20 \%$ do extrato líquido da casca da aroeira, álcool de cereais (q.b.) e 0,01g de conservantes.

Foram realizados 94 exames clínicos em pacientes usuários de próteses mucossuportadas superiores atendidos na Clínica Protética da Universidade Federal da Paraíba e no Centro Odontológico de Cruz das Armas (COCA), na cidade de João Pessoa, Paraíba, Brasil. Para participar do estudo, os pacientes assinaram o Termo 
de Consentimento Livre e Esclarecido. Fizeram parte da amostra pacientes de ambos os sexos e com diagnóstico clínico para Estomatite Protética Tipo II no palato ${ }^{11}$ e presença de candidose associada, diagnosticada através de exame micológico. Os pacientes não podiam apresentar nenhum fator predisponente de ordem sistêmica para o aparecimento da infecção, como diabetes, hiperparatireóidismo, imunodeficiências e tratamentos radioterápicos ou quimioterápicos, como também não estar fazendo uso de medicamentos que predispusessem à infecção por Candida spp.

O material biológico foi coletado da base da prótese e da mucosa palatina sendo em seguida semeados em placas de Petri contendo o meio de cultura Ágar Sabouraud Dextrose (DIFCO $\left.{ }^{\circledR}\right)$ acrescido de clorafenicol $(0,1 \mathrm{mg} / \mathrm{ml})$. As placas de Petri foram encaminhadas ao Laboratório de Micologia da UFPB, acondicionadas em recipiente com tampa, obedecendo as normas de biossegurança, dentro de um período de duas horas, sendo incubadas em estufa a 37 으 por um período de 24-72 horas para posterior processamento.

Foi realizada a contagem de UFC/mL de Candida spp. e a identificação das espécies através da observação de características morfológicas macro e microscópicas (tubo germinativo, pseudohifas e clamidósporos) e provas bioquímicas (auxanograma e zimograma). O diagnóstico de candidose estava condicionado a presença de UFC/mL acima de 20. Uma contagem com valor abaixo do citado acima foi considerado como colonização ${ }^{3}$.

A amostra final foi composta por 18 indivíduos de ambos os sexos, com idade variando entre 36 a 75 anos (média de 55,64 anos). Todos os pacientes que participaram do ensaio clínico tiveram diagnóstico clínico para estomatite protética tipo $\mathrm{II}^{11}$. Os demais pacientes foram excluídos da amostra por desistência ou por não obedecerem às orientações prescritas. Os pacientes foram questionados quanto à forma e freqüência de higienização da prótese. Todos afirmaram realizar a higiene da prótese através da escovação com dentifrício, três vezes ao dia. Os pacientes foram divididos em dois grupos: Grupo Teste (GT): nove integrantes que fizeram uso do spray de aroeira; Grupo Controle (GC): nove integrantes que fizeram uso de Nistatina suspensão oral ${ }^{\circledR}$ em forma de Spray (Controle Positivo).

Os pacientes foram orientados a realizar a higienização rotineira da prótese através da escovação com dentifrício, excluindo-se o uso de qualquer tipo de agente químico, e em seguida realizar o borrifamento do produto na mucosa palatal e na base da prótese três vezes ao dia durante quinze dias consecutivos. Após o borrifamento os pacientes deveriam utilizar a prótese como de costume e retirá-la ao dormir armazenando-a em um copo com água.

Para avaliação da eficácia do tratamento, foi realizado um segundo exame clínico e micológico no 15 dia de uso dos produtos, sendo feita uma classificação do tratamento através de dois parâmetros. O primeiro parâmetro foi uma análise clínica, onde o tratamento foi considerado satisfatório quando houvesse remissão total do processo inflamatório no palato, regular quando houvesse remissão parcial da inflamação, ou insatisfatório quando não houvessem alterações no processo inflamatório do palato. O segundo parâmetro foi a análise micológica, onde o tratamento foi considerado satisfatório quando houvesse ausência de infecção fúngica no palato ou na prótese, regular quando houvesse uma diminuição nas $\mathrm{UFC} / \mathrm{mL}$ mas permanecesse a infecção, e insatisfatório quando não houvessem alterações nas UFC/mL.

Os resultados foram avaliados pelos testes de Wilcoxon e Mann-Whitney, com significância de 95\%, utilizando-se o programa GMC versão 8.2.

\section{RESULTADOS}

Os resultados referentes ao ensaio clínico estão expressos nas Tabelas 1 e 2. Observou-se uma redução estatisticamente significante para GC e GT pelo teste de Wilcoxon, com $\mathrm{p}=0,01$, quanto à análise clínica e micológica. Ao aplicar-se o teste estatístico de MannWhitney, observou-se que não houve diferença estatisticamente significante entre GT e GC $(p>0,05)$.

Tabela 1. Resultados da análise clínica de acordo com a classificação proposta e grupos experimentais.

\begin{tabular}{cccccccc}
\hline \multirow{2}{*}{ Grupo } & \multicolumn{2}{c}{ Satisfatório } & \multicolumn{2}{c}{ Regular } & \multicolumn{2}{c}{ Insatisfatório } & \multirow{2}{*}{ Total } \\
& $\mathrm{n}$ & $\%$ & $\mathrm{n}$ & $\%$ & $\mathrm{n}$ & $\%$ & \\
\hline GT & 6 & 66,7 & 3 & 33,3 & 0 & 0 & 9 \\
GC & 7 & 77,8 & 2 & 22,2 & 0 & 0 & 9 \\
\hline
\end{tabular}

Tabela 2. Resultados da análise micológica de acordo com a classificação proposta e grupos experimentais.

\begin{tabular}{cccccccc}
\hline \multirow{2}{*}{ Grupo } & \multicolumn{2}{c}{ Satisfatório } & \multicolumn{2}{c}{ Regular } & \multicolumn{2}{c}{ Insatisfatório } & \multirow{2}{*}{ Total } \\
& $\mathrm{n}$ & $\%$ & $\mathrm{n}$ & $\%$ & $\mathrm{n}$ & $\%$ & \\
\hline GT & 7 & 77,8 & 1 & 11,1 & 1 & 11,1 & 9 \\
GC & 8 & 88,9 & 1 & 11,1 & 0 & 0 & 9 \\
\hline
\end{tabular}

A C. albicans foi o microorganismo mais prevalente, estando presente em $94,4 \%$ dos casos, sendo que em $77,8 \%$ dos casos ela esteve presente de forma isolada, em $11 \%$ associada à $C$. parapsilosis, em 5,6\% associada à C. krusei. Um único caso o microorganismo identificado 
foi a C. tropicalis (5,6\%) (Tabela 3). Em todos os casos, a infecção fúngica foi diagnosticada apenas nas amostras coletadas da superfície interna da prótese.

Tabela 3. Espécies de Candida identificadas de acordo com o número de casos e a porcentagem.

\begin{tabular}{ccc}
\hline Espécie de Candida & \multicolumn{3}{c}{ Frequência } \\
& $\mathrm{n}$ & $\%$ \\
\hline C. albicans & 14 & 77,8 \\
C. albicans + C. parapsilosis & 2 & 11 \\
C. albicans + C. krusei & 1 & 5,6 \\
C. tropicalis & 1 & 5,6 \\
\hline
\end{tabular}

\section{DISCUSSÃO}

Definidacomoinfecçãoda cavidadebucal por Candida spp., a candidose bucal é considerada a infecção fúngica mais comum entre humanos. As espécies desse fungo são freqüentemente isoladas da cavidade bucal, chegando a estar presente em $40 \%$ dos indivíduos de uma população. No entanto, diante de alterações como redução do fluxo salivar, trauma local, mudanças de pH e uso contínuo de próteses removíveis, esses microrganismos podem atuar como patógenos oportunistas, desencadeando processos infecciosos ${ }^{12}$.

No presente estudo foi realizado um exame micológico para avaliar a presença de infecção por Candida spp. em todos os pacientes que se submeteram ao ensaio clínico, sendo realizada coleta de material biológico proveniente da mucosa palatina subjacente à prótese, como também da superfície interna da prótese. A C. albicans foi o microorganismo mais prevalente, estando presente em $94,4 \%$ dos casos. Entretanto outros microorganismos foram identificados, como a C. parapsilosis (11\%), a C. krusei $(5,6 \%)$ e a C. tropicalis (5,6\%). Diversos estudos demonstram que a $C$. albicans é a espécie mais comumente encontrada em indivíduos com estomatite protética ${ }^{1,2,13,14}$, sendo responsável por aproximadamente $70 \%$ dos casos de infecção, entretanto, uma variedade de espécies não-albicans, como $C$. krusei, C. glabrata, C. tropicalis e C. dubliniensis tem sido relacionada a um grande número de infecções ${ }^{1}$.

No presente estudo, a infecção por Candida spp. foi diagnosticada, em todos os casos, apenas na superfície interna da prótese. Este fato está de acordo com a literatura, onde estudos têm demonstrado que as colônias de Candida spp. são isoladas, mais freqüentemente, da superfície interna das próteses quando comparadas à mucosa correspondente ${ }^{15}$, sendo encontradas em torno de $80 \%$ das próteses ${ }^{7,16}$. $\mathrm{O}$ ambiente sub prótese proporciona um meio propício para a adesão dos microorganismos, pois há ausência de saliva impossibilitando a ação de substâncias como a amilase e $\lg A^{5,6}$. O local preferencial de adesão desses microorganismos são as rachaduras e irregularidades da prótese, passando a atuar como reservatório para disseminação crônica de microorganismos. Desta forma, o tratamento da estomatite protética deve, necessariamente, envolver cuidados de higienização e desinfecção das próteses dentárias ${ }^{5}$.

Diversos medicamentos têm sido indicados para o tratamento da estomatite protética, podendo ser de aplicação tópica e sistêmica. Na terapia tópica, antifúngicos como nistatina, miconazol e clorexidina têm sido indicados. Apesar de esses medicamentos serem eficazes para aliviar os sinais e sintomas clínicos desta lesão, muitas vezes não eliminam completamente o microorganismo, levando a infecção recorrente e, consequentemente, ao insucesso no tratamento ${ }^{13}$. Além disso, uma segunda desvantagem na utilização destes antifúngicos é o aparecimento de microorganismos resistentes durante tratamento a longo prazo, o qual tem sido apontado como uma das principais causas do insucesso desse tipo de terapia ${ }^{17}$. Atualmente, diversos estudos têm sido realizados para avaliar a eficácia de produtos fitoterápicos no tratamento de infecções, inclusive da estomatite protética ${ }^{18,19}$. A facilidade de acesso, o baixo custo e a compatibilidade cultural tem tornado a fitoterapia um recurso terapêtico recomendável especialmente para comunidades carentes, cuja assistência é difícil, como é o caso da região Nordeste ${ }^{10}$.

Portanto, neste estudo foi avaliada a eficácia da utilização de uma tintura da casca da aroeira (Schinus terebinthifolius Raddi) no tratamento da estomatite protética, sendo avaliada a remissão do processo inflamatório presente no palato, bem como a eliminação da infecção por espécies de Candida presentes na base da prótese dos pacientes. Os resultados demonstraram que o tratamento instituído com esta planta, aplicada três vezes ao dia durante 14 dias consecutivos, resultou em completa eliminação das alterações clínicas inflamatórias do palato dos pacientes em $66,7 \%$ dos casos e em eliminação parcial da inflamação em 33,3\% dos casos. Em nenhum paciente o tratamento foi considerado insatisfatório.

Diversos estudos demonstraram que a casca da aroeira apresenta elevado poder antiinflamatório e cicatrizante. Pesquisa prévia avaliou a eficácia do extrato hidroalcoólico da casca da aroeira no tratamento da alveolite induzida em ratos, observando que esta planta estimulou o processo de cicatrização e neoformação óssea, apresentando resultados superiores ao controle positivo ${ }^{20}$. Este fato também foi observado em lesões 
ulceradas induzidas em ratos, onde o extrato da casca desta planta atuou acelerando o processo de reparo do tecido epitelial e conjuntivo, estimulando a ceratinização e diminuindo rapidamente a intensidade do processo inflamatório ${ }^{21,22}$.

No presente estudo foi observado ainda que houve eliminação da infecção fúngica presente na superfície interna da prótese em $77,7 \%$ dos pacientes tratados com a S. terebinthifolius. Os microorganismos presentes nestes pacientes foram $C$. albicans, $C$. parapsilosis e $C$. krusei. A atividade antifúngica da casca da aroeira frente à C. albicans foi observada em diversos estudos, um dos quais constaou que o extrato etanólico apresentou atividade antifúngica frente à $C$. albicans na concentração de $1 \%$, com halo de inibição de $11,9 \mathrm{~mm}$, pela técnica da Concentração Inibitória Mínima (CIM) ${ }^{23}$. Outra pesquisa in vitro demonstrou que o extrato aquoso apresentou elevada atividade antifúngica sobre $C$. albicans, com CIM igual a $120 \mathrm{ng} / \mathrm{ml}$, apresentando resultados superiores a Anfotericina $B$, que obteve CIM igual a 950ng/m/24.

No atual estudo em apenas um caso o tratamento com a aroeira foi considerado insatisfatório considerandose o aspecto eliminação da infecção fúngica. Neste caso, a espécie diagnosticada foi a $C$. tropicalis. Pesquisa prévia revelou que o extrato da casca da aroeira apresentou atividade frente à C. albicans, com CIM de $1250 \mu \mathrm{g} / \mathrm{mL}$, entretanto o extrato não apresentou ação frente à $C$. tropicalis ${ }^{25}$. Isto demonstra que esta espécie de Candida parece ser mais resistente à terapia com antimicrobianos, o que pode estar relacionado ao insucesso do caso no presente estudo.

Na presente pesquisa, a parte da planta escolhida foi a casca, devido ao fato da maioria dos estudos que comprovaram a atividade antimicrobiana e antiinflamatória terem sidos realizados com esta parte da planta. A casca do S. terebenthifolius apresenta diversos compostos químicos na sua constituição, como flavonóides, taninos e triterpenos. Estes compostos são considerados responsáveis pelas atividades antibacteriana, antifúngica e antiinflamatória, o que confere largo uso desta planta no tratamento de doenças infecciosas e inflamatórias ${ }^{9}$. Os taninos apresentam a capacidade de precipitar proteínas das células superficiais de mucosas e tecidos, formando uma camada protetora (complexo tanino/proteína) sobre a pele ou mucosa danificada, apresentando também elevada atividade antimicrobiana ${ }^{26}$. Os flavonóides apresentam comprovada atividade antiinflamatória e antioxidante ${ }^{27}$ e os triterpenos e apresentam atividade antiinflamatória, por atuarem como inibidores competitivos da fosfolipase $\mathrm{A} 2^{28}$.

A tintura da aroeira foi realizada de acordo com a técnica da maceração. Para tanto, foi utilizado o álcool de cereais como líquido extrator. Este álcool é bastante utilizado na formulação de tinturas fitoterápicas e estudos in vitro demonstram ausência ${ }^{29}$ ou apenas discreta atividade antimicrobiana deste álcool ${ }^{30}$. Desta forma, pode-se pressupor que os resultados da atividade antifúngica e antiinflamatória observados na presente pesquisa é proveniente dos compostos químicos presentes na casca da aroeira.

Com relação aos resultados referentes à nistatina, pode-seobservar reduçãoclínica emicológica satisfatórias, sendo este resultado estatisticamente significante. Este resultado difere dos estudos encontrados na literatura, sendo que em um deles, com 17 pacientes com diagnóstico clínico e micológico para estomatite protética, a terapêutica com este medicamento realizada durante 15 dias consecutivos resultou na cura clínica em $57 \%$ das lesões e na eliminação da infecção fúngica em $44 \%$ dos $\operatorname{casos}^{19}$. Em outra pesquisa, pacientes com diagnóstico clínico e micológico para estomatite protética utilizaram a nistatina durante 15 dias consecutivos resultando na eliminação da infecção em apenas $20 \%$ dos $\operatorname{casos}^{18}$. Este resultado pode ser devido ao fato de que, no presente estudo, a nistatina foi utilizada na forma de spray, sendo aplicada diretamente na mucosa palatal e na base da prótese, o que proporcionou um maior tempo de contato do produto com a área infectada.

Desta forma, o presente estudo demonstrou que a casca da S. terebinthifolius foi eficaz no tratamento da estomatite protética, proporcionando eliminação da infecção fúngica da superfície interna da prótese, bem como remissão do processo inflamatório presente na mucosa palatina, apresentando resultados semelhantes à terapia convencional. Entretanto, são necessários mais estudos que avaliem o sucesso do tratamento com esta planta a longo prazo.

\section{CONCLUSÃO}

A utilização da tintura da casca da aroeira é uma alternativa terapêutica eficaz no tratamento da estomatite protética, promovendo remissão dos sinais clínicos e eliminação da infecção por Candida spp. presente na prótese.

\section{REFEREANCIAS}

1. Webb BC, Thomas CJ, Whittle T. A 2-year study of Candidaassociated denture stomatitis treatment in aged care subjects. Gerodontology 2005; 22(3):168-76. 
2. Golecka M, Oldakowska-Jedynak U, Mierzwinska-Nastalska E, Adamczyk-Sosinska E. Candida-associated denture stomatitis in patients after immunosuppression therapy. Transplant Proc 2006; 38(1):155-6.

3. Budtz-Jorgensen E, Mojon P, Banon-Clement JM, Baehni P. Oral candidosis in long-term hospital care: comparison of edentulous and dentate subjects. Oral Dis 1996; 2(4):285-90.

4. Campos MS, Marchini L, Bernardes LA, Paulino LC, Nobrega FG. Biofilm microbial communities of denture stomatitis. Oral Microbiol Immunol 2008; 23(5):419-24.

5. Maza JL, Elzeguebal N, Prado C, Ellacuria J, Soler I, Ponton J. Candida albicans adherence to resin-composite restorative dental material: Influence of whole human saliva, Oral Surg Oral Med Oral Pathol Oral Radiol Endod 2002; 94(5):589-92.

6. Ramage G, Tomsett K, Wickes BL, Lópes-Ribot JL, Redding SW. Denture stomatitis: a role for Candida biofilms. Oral Surg Oral Med Oral Pathol Oral Radiol Endod 2004; 98(1):53-9.

7. Lemos MMC, Miranda JL, Souza MSGS. Estudo clínico, microbiológico e histopatólogico da estomatite por dentadura. Rev Bras Patol Oral 2003; 2(1):3-10.

8. Lombardi T, Budtz-Jörgensen E. Treatment of dentureinduced stomatitis: a review. Eur J Prosthodont Restor Dent 1993; 2(1):17-22.

9. Lima MRF, Luna JS, Santos AF, Andrade MCC, Sant'Ana AEG, Genet JP, Marquez B, Neuville L, Moreau N. Anti-bacterial activity of some brazilian medicinal plants. J Ethnopharmacol 2006; 105(1-2):137-47.

10. Lorenzi $\mathrm{H}$, Matos FJA. Plantas medicinais no Brasil: nativas e exóticas. São Paulo: Instituto Plantarum, 2002. 544p.

11. Newton AV. Denture sore mouth. A possible aetiology. $\mathrm{Br}$ Dent J 1962; 102(1):357-60.

12. Calderone RA, Fonzi WA. Virulence factors of Candida albicans. Trends Microbiol 2001; 9(7):327-35.

13. Banting DW, Hill SA. Microwave disinfection of dentures for the treatment of oral candidiasis. Spec Care Dentist 2001; 21(1):4-8.

14. Luo G, Samaranayake LP. Candida glabrata, an emerging fungal pathogen, exhibits superior relative cell surface hydrophobicity and adhesion to denture acrylic surfaces compared with Candida albicans. APMIS 2002; 110(9):601-10.

15. Monsenego P. Presence of microorganisms on the fitting denture complete surface: study "in vivo". J Oral Rehabil 2000; 27(8):708-13.

16. Pardi G, Cardozo El, Perrone M, Salazar E. Detección de especies de Candida en pacientes con estomatitis subprotetica. Acta Odontol Venezol 2001; 39(3):32-44.

17. Goldman GH, Ferreira MES, Marques ER, Savoldi M, Perlin D, Park S, Godoy Martinez PC, Goldman MH, Colombo AL. Evaluation of fluconazole resistance mechanisms in candida albicans clinical isolates from HIV-infected patients in Brazil. Diagn Microbiol Infect Dis 2004; 50(1):25-32.

18. Xavier MN. Terapêutica alopática x fitoterápica em pacientes idosos portadores de candidose bucal. Manifestações bucais na senescência. [Dissertação]. João Pessoa: Faculdade de Odontologia, Universidade Federal da Paraíba; 1997.

19. Santos RC. Identificação do gênero Candida na cavidade bucal de usuários de prótese dentária e avaliação terapêutica do fitoterápico a base de Cymbopogon citratus. [Dissertação]. João Pessoa: Faculdade de Odontologia, Universidade Federal da Paraíba; 2000.

20. Melo Júnior EJ, Raposo MJ, Lisboa Neto JA, Diniz MF, Marcelino Júnior CA, Sant'Ana AE. Medicinal plants in the healing of dry socket in rats: microbiological and microscopic analysis. Phytomedicine 2002; 9(2):109-16.

21. Coutinho IHILS, Torres OJM, Matias JEF, Coelho JCU, Stahlke Júnior HJ, Agulham MA et al. Efeito do extrato hidroalcoólico de aroeira (Schinus terebinthifolius Raddi) na cicatrização de anastomoses colônicas. Estudo experimental em ratos. Acta Cir Bras 2006; 21(Supp 3):49-54.

22. Ribas MO, Souza ME, Sartoreto J, Lanzoni TA, Noronha L, Acra LA. Efeito da Schinus terebinthifolius Raddi sobre o processo de reparo tecidual das lesões ulceradas induzidas na mucosa bucal do rato. Rev Odonto Ciênc 2006; 21(53):245-52.

23. Guerra MJM, Barreiro ML, Rodrígues ZM, Rubalcaba Y. Actividad antimicrobiana de un extracto fluido al $80 \%$ de Schinus terebinthifolius raddi (copal). Rev Cuba Plantas Med 2000; 5(1):23-5.

24. Schmourlo G, Mendonça-Filho RR, Alviano CS, Costa SS. Screening of antifungal agents using ethanol precipitation and bioautography of medicinal and food plants. J Ethnopharmacology 2005; 96(3):563-8.

25. Lima EO, Pereira FO, Lima IO, Trajano VN, Souza EL. Schinus terebenthifolius Raddi: avaliação do espectro de ação antimicrobiana do seu extrato aquoso. Infarma 2004; 16(7):83-5.

26. Haslam E. Natual polyphenols (vegetable tannins) as drugs and medicine: possible modes of action. J Nat Prod 1996; 59(2):205-15.

27. Velázquez E, Tournier HA, Mordujovich de Buschiazzo P, Saavedra G, Schinella GR. Antioxidant activity of Paraguayan plant extracts. Fitoterapia 2003; 74(1-2):91-7.

28. Jain MK, Yu BZ, Rogers JM, Smith AE, Boger ET, Ostrander $\mathrm{RL}$, Rheingold AL. Specific competitive inhibitor of secreted phospholipase A2 from berries of Schinus terebinthifolius. Phytochemistry 1995; 39(3):537-47.

29. Silva NB, Claudinho LV, Neves AS, Costa AC, Valença AMG. Avaliação da atividade antimicrobiana de tinturas fitoterápicas sobre porphyromonas gigivalis e prevotella melaninogenica. Pesq Bras Odontoped Clin Integr 2006; 6(2):167-71.

30. Matos BM, Komiyama EY, Balducci I, Koga-Ito CY. Atividade antifúngica do extrato alcoólico de Mentha piperita sobre. Candida albicans e C. tropicalis. Rev Odontol UNESP 2009; 38(4):244-8.

Recebido/Received: 19/01/10 Revisado/Reviewed: 13/06/10 Aprovado/Approved: 29/06/10

Correspondência:

Diana Gabriela de Sousa Soares

Av. Duque de Caxias, 942 - Centro

Araraquara/SP

CEP: $14801-120$

Telefones: (16) 3322-0732; (16) 8814-0517

E-mail: diana_odonto@yahoo.com.br 